\title{
Tabagismo entre trabalhadores de empresa bancária*
}

\section{Cigarette smoking among workers of a Bank}

\section{Rosane H. G riep, Dora Chór e Luiz A. B. Camacho}

Departamento de Enfermagem em Saúde Pública da Escola de Enfermagem Anna Nery da Universidade Federal do Rio de Janeiro. Rio de Janeiro, RJ - Brasil (R.H.G.), Escola Nacional de Saúde Pública da Fundação O swaldo Cruz. Rio de Janeiro, RJ - Brasil (D.C., L.A. B.C.)

G RIEP Rosane H., Dora Chór e Luiz A. B. Camacho Tabagismo entre trabalhadores de empresa bancária* Rev. Saúde Pública, 32 (6): 533-40, 1998

(c) Copyright Faculdade de Saúde Pública da USP. Proibida a reprodução mesmo que parcial sem a devida autorização do Editor Cient́fico. Proibida a utilização de matérias para fins comerciais. All rights reserved. 


\title{
Tabagismo entre trabalhadores de empresa bancária*
}

\section{Cigarette smoking among workers of a Bank}

\author{
Rosane H. Griep, Dora Chór e Luiz A. B. Camacho \\ Departamento de Enfermagem em Saúde Pública da Escola de Enfermagem Anna Nery \\ da Universidade Federal do Rio de Janeiro. Rio de Janeiro, RJ - Brasil (R.H.G.), Escola N acional \\ de Saúde Pública da Fundação O swaldo Cruz. Rio de Janeiro, RJ - Brasil (D.C., L.A. B.C.)
}

\begin{abstract}
Resumo
Objetivo Descrever a prevalência de tabagismo e sua associação com outros fatores de risco para doenças crônicas entre funcionários dos centros de processamentos de serviços e comunicações de uma empresa bancária.

Método Estudo seccional de amostra aleatória simples de 647 funcionários, através de questionário auto-respondido no ambiente de trabalho.

Resultados A prevalência de tabagismo foi de 29,5\% (Intervalo de Confiança (IC) $95 \%$ : $27,5 \%$ a $31,5 \%$ ), sendo $31,1 \%$ (IC $95 \%: 26,2 \%$ a $35,8 \%$ ) entre homens e $27,8 \%$ (IC 95\%: 22,6\% a 32,9\%) entre mulheres. O início do hábito ocorreu, em média, aos 17,6 anos entre os homens e 19,4 anos entre as mulheres. Observou-se alta prevalência de grandes fumantes entre homens e mulheres (53\% e $42 \%$ respectivamente fumavam mais de 20 cigarros por dia). A frequiência de tabagismo foi maior nos mais velhos, nos divorciados separados e viúvos, nos hipertensos, naqueles que consumiam mais bebidas alcoólicas, e nos que não praticavam exercícios físicos. Comparados aos não-fumantes, os ex-fumantes eram mais velhos, consumiam mais bebidas alcoólicas e apresentavam maior freqüência de sobrepeso.

Conclusão A frequiência de tabagismo e de outros fatores de risco para as doenças crônicas, nesta categoria de trabalhadores, aponta para a necessidade de repensar estratégias das ações de saúde atualmente desenvolvidas. Oportunidades de intervenções preventivas mais eficazes e de menor custo podem estar sendo perdidas.
\end{abstract}

Tabagismo, epidemiologia. Fatores de risco. Trabalhadores.

\section{Abstract}

Objective To describe the prevalence of cigarette smoking and its association with other risk factors for chronic diseases among active workers of communication and data processing centers of a Bank.

Methods Cross-sectional study in a simple random sample of 647 active workers of the bank. The data were collected in the work environment, through self-administered questionnaires.

*Baseado em dissertação de mestrado apresentada à Escola Nacional de Saúde Pública, 1996.

Correspondência para/Correspondence to: Rosane H. Griep - Rua Afonso Cavalcanti, 275 - Cidade Nova -20211-110 Rio de Janeiro, RJ - Brasil. Recebido em 10.12.1997. Reapresentado em .8.4.1998. Aprovado em .16.4.1998. 
Results The prevalence of cigarette smoking was $29.5 \%(95 \%$ Confidence Interval (CI): $27.5 \%-31.5 \%), 31.1 \%$ (95\%CI: $26.2 \%-35.8 \%$ ) among men and $27.8 \%$ (95\% CI: 22.6\%-32.9\%) among women. On average, males started smoking at the age of 17.6 years and women at the age of 19.4. High prevalence of heavy smokers was observed among men and women (53\% and 42\%, respectively, smoked more than 20 cigarettes per day). Smokers were older, more likely to be divorced, separated and widowed, to have high blood pressure, to drink alcoholic beverages more often, and to exercise less often than to non-smokers. Those who gave up smoking were older, drank more alcoholic beverages, and were more often overweight.

Conclusion

The considerable frequency of smoking and other risk factors for chronic diseases among those workers may be an indication of the need for new strategies for health interventions. Opportunities for preventive actions, which are more effective and less costly, may have been lost.

Smoking, epidemiology. Risk factors. Workers.

\section{INTRO D U ÇÃO}

Muitos estudos epidemiológicos têm associado hábitos e comportamentos a diversas doenças crônicas, tais como, a doença isquêmica do coração e vários tipos de neoplasias. O sedentarismo, o excessivo consumo de gorduras saturadas e baixo consumo de vegetais e especialmente o fumo estão entre estes hábitos (Epstein ${ }^{10}$,1989; Doll e Hill ${ }^{7}, 1956$; Kabat $^{22}$, 1994). Há comprovadas evidências do efeito doseresposta do uso do tabaco e o incremento de doenças (Serxner e col. ${ }^{36}, 1992$ ), sendo que três fatores influenciam diretamente no aumento do risco de adoecer: a precocidade do início do uso, a quantidade de cigarros fumados ao dia e a profundidade da tragada (Fielding $\left.{ }^{13}, 1986\right)$. Estudos mais recentes têm apontado ainda o aumento do risco de adoecer entre fumantes passivos, que apresentam maior incidência de doenças respiratórias na infância (Ronchetti e col. $^{34}, 1990$; Price $^{30}$, 1990; Rosemberg ${ }^{35}$, 1987). Baixo peso ao nascer de filhos de grávidas fumantes também tem sido referido em alguns estudos (Hoppenbrouwers ${ }^{18}, 1990$; Ronchetti e col. ${ }^{34}, 1990$; Taylor $^{39}, 1990$ ). Além disso, entre adultos expostos à fumaça do cigarro, a precipitação ou agravamento de problemas alérgicos, angina de peito, e também o risco de desenvolver câncer de pulmão têm sido mais freqüentes do que entre adultos não expostos (Joosens ${ }^{20}$, 1990; Ronchetti e col. $\left..^{34}, 1990\right)$.

Em função deste quadro, o combate ao consumo do tabaco tem sido priorizado na maioria dos países desenvolvidos, através dos mais diversos programas de intervenção, que visam a reduzir a prevalência de fumantes, como também a prevenção do uso do cigarro entre crianças e adolescentes (Puska e col. ${ }^{31}$, 1983; Cawston e McEwen ${ }^{4}$, 1994; Osler ${ }^{29}$, 1993; Baan $^{1}$, 1990).
Uma das estratégias frequientemente utilizadas para alcançar este objetivo, tem sido a implantação de programas de promoção da saúde nas empresas. $\mathrm{O}$ ambiente de trabalho tem sido apontado como um local favorável para modificar hábitos, já que nestes, os funcionários passam a maior parte do dia, proporcionando excelentes oportunidades para ações educativas, o que permite reduzir assim, o número de absenteísmo e gastos com doenças (Fielding e col. ${ }^{12}$, 1990). A maioria das empresas de países desenvolvidos criaram legislação específica proibindo ou restringindo o uso de cigarros em locais coletivos (Roemer ${ }^{33}$, 1993; Joossens ${ }^{20}, 1990$; Fielding e col. ${ }^{11}$,1993). Estas medidas constituem conquistas importantes para os não-fumantes e têm estimulado o abandono ao hábito de fumar, bem como a redução do número de cigarros fumados entre os trabalhadores (Wakefield e col. ${ }^{41}, 1992$, Jeferry e col. ${ }^{19}$, 1994).

No Brasil, as informações a respeito da prevalência de tabagismo são ainda insuficientes, apesar de sua importância para a saúde pública. A maior parte dos estudos dizem respeito à década de 80 , e no mais recente, realizado pelo IBGE $^{15}$ (1989), estimou-se em 32,6\% a prevalência de fumantes no País. Apesar de termos avançado nos últimos anos no que se refere ao desenvolvimento de leis anti-tabaco, as dificuldades de se fazer cumprir estas leis são ainda bastante evidentes. Além disso, campanhas de prevenção do hábito de fumar entre os jovens são ainda incipientes e pontuais (Ministério da Saúde ${ }^{25}$,1992).

A interrupção abrupta do consumo de tabaco pode desencadear síndrome de abstinência, caracterizada por irritabilidade, agressividade, hostilidade, depressão e ânsia de fumar entre grandes fumantes (Fielding e col. ${ }^{11}$, 1993; Kavanagh e col. ${ }^{23}$, 1993; Daughton e col. $^{6}, 1990$; Brigham e col. $\left.{ }^{3}, 1994\right)$. O reconhecimen- 
to do tabagismo como uma forma de dependência implicou necessidade de dar suporte aos indivíduos que manifestam vontade de largar o tabaco, bem como de reforçar a necessidade de evitar que o hábito seja adquirido.

O presente estudo estima a prevalência do tabagismo entre funcionários dos centros de processamentos de serviços e comunicações (CESECs) de uma empresa bancária, e avalia também a associação do hábito de fumar com outros fatores de risco, variáveis socioeconômicas, biológicas e de perfil funcional, a fim de contribuir para a implementação de programas de promoção da saúde que busquem modificar o estilo de vida e as condições de saúde dos funcionários.

\section{MÉTODO}

Foi realizado estudo seccional em amostra aleatória simples dos funcionários dos CESECs de uma empresa bancária no Estado do Rio de Janeiro, entre agosto a dezembro de 1994. A população-alvo foi constituída por funcionários ativos das carreiras técnicas (funcionários de nível superior, que exercem suas atividades, como médicos, engenheiros e advogados) e administrativas (bancários propriamente dito). A única carreira excluída foi a de apoio, pelo reduzido número de funcionários na empresa e também, não se exigia escolaridade mínima para sua admissão, o que dificultaria o preenchimento do questionário.

Utilizou-se questionário auto-respondido, no ambiente de trabalho, com objetivo de minimizar tempo e custos, já que o alto grau de escolaridade dos funcionários permitia tal escolha. Antes do preenchimento, os funcionários recebiam um termo de consentimento que esclarecia sobre a finalidade da pesquisa e ressaltava o caráter voluntário da participação e da identificação. Além deste documento, aplicadores treinados enfatizavam a importância do preenchimento e estavam aptos a esclarecer dúvidas.

\section{Variáveis}

As variáveis e suas categorias consideradas para a análise foram:

Tabagismo: a) fumantes - uso atual de cigarros por algum indivíduo que fumou, ao longo da vida, pelo menos cinco carteiras de cigarros; $b$ ) ex-fumantes - indivíduos que se classificaram como ex-fumantes; c) não-fumantes - indivíduos que nunca fumaram, ou que fumaram menos de cinco carteiras de cigarro ao longo da vida. A classificação de hipertensão arterial foi realizada a partir de quatro perguntas do questionário, nos quais o respondente informava se havia feito tratamento, se havia sido informado por profissional de saúde sobre sua condição de hipertenso, qual era sua pressão arterial habitual e se já havia tido ou tinha no momento hipertensão arterial. Com base em algoritmo construído a partir destes itens, os funcionários foram classificados como hipertensos ou normotensos. Assim, por exemplo, de acordo com o critério número 1 , se o funcionário respondeu que já havia sido informado mais de uma vez, em dias diferentes, de que era hipertenso e que fez tratamento no passado, foi classificado como portador da enfermidade.

Índice de massa corporal (IMC $=$ peso/altura ${ }^{2}$ ): a) baixo peso: $\mathrm{IMC}<20 \mathrm{~kg} / \mathrm{m}^{2}$; b) normal: $20 \mathrm{~kg} / \mathrm{m}^{2} \leq \mathrm{IMC} \leq$ $24,9 \mathrm{~kg} / \mathrm{m}^{2}$; c) sobrepeso: $25 \mathrm{~kg} / \mathrm{m}^{2} \leq \mathrm{IMC} \leq 30 \mathrm{~kg} / \mathrm{m}^{2}$; d) obeso: $\mathrm{IMC}>30 \mathrm{~kg} / \mathrm{m}^{2}$.

Atividade física regular: praticar atividades físicas específicas para melhorar a saúde, condicionamento físico ou para fins estéticos pelo menos duas vezes por semana, durante pelo menos vinte minutos cada vez.

Cosnsumo de álcool: a) não bebe - para os funcionários que responderam as opções: "parei de beber" ou "nunca tomei bebida alcoólica"; b) bebe ocasionalmente - para os que responderam as opções: "ocasionalmente" (pelo menos uma vez por mês) ou "raramente" (menos de uma vez por mês); c) bebe freqüentemente - para os que responderam as opções: beber "diariamente" ou "quase todo dia" (quatro ou mais vezes por semana) ou "pelo menos uma vez por semana"(até três vezes por semana).

Informado sobre níveis de colesterol sangüíneo: para os indivíduos que responderam sim à pergunta: "Algum médico ou outro profissional da área da saúde já lhe disse que seus níveis de colesterol no sangue estavam elevados?"

Horário de trabalho a) noturno: para os funcionários que entram no banco nos horários de 19 às 6 horas: $b$ ) diurno: para os que entram de 7 às 18 horas.

\section{Análise Estatística}

As principais variáveis estudadas foram apresentadas através das freqüências absolutas e proporções, com seus respectivos intervalos (IC) com nível de significância de 95\%. Para avaliar diferenças estatísticas entre proporções utilizou-se o Qui-quadrado $\left(\mathrm{c}^{2}\right)$, e entre médias, a análise de variância (ANOVA) ou teste $\mathbf{t}$ de Student. Para melhor avaliar a relação das variáveis estudadas com o tabagismo e ainda estudar o efeito de cada variável ajustada pelas demais, utilizou-se a técnica de regressão logística. Dois modelos foram testados: o primeiro, teve como categorias da variável dependente "não-fumantes" $(0)$ e "fumantes" (1); no segundo, "não-fumantes"(0) e "ex-fumantes" (1). A seleção das variáveis foi feita a partir das associações detectadas na análise bivariada anterior, exceto idade e sexo que foram "forçadas" no modelo, fazendo prevalecer critérios lógicos sobre critérios estatísticos.

\section{RESU LTAD OS}

A pesquisa teve alta adesão por parte dos funcionários, já que $97 \%$ dos sorteados responderam ao questionário. 
A idade variou entre 22 e 59 anos, com média de 37,9 anos e mediana de 38 anos. As mulheres eram mais velhas, com idade média de 39 anos, enquanto entre os homens esta medida foi de 36,9 anos ( $\mathrm{p}<0,001)$. A renda per capita média foi de $\mathrm{R} \$ 348,40$, mediana e moda de $\mathrm{R} \$ 300,00 ; 60 \%$ eram proprietários do imóvel onde residiam e a maior parte era casada $(60 \%)$. Destaca-se o alto grau de escolaridade apresentada pelos funcionários, já que $86 \%$ chegaram a ingressar na universidade (Tabela 1).

Nos CESECs, 99,2\% dos funcionários pertenciam ao quadro administrativo, 75,3\% trabalhavam durante o dia, e pela natureza das tarefas realizadas (compensação de cheques e outras atividades inter-

Tabela 1 - Características socioeconômico e demográficas da população estudada, 1994.

\begin{tabular}{llrc}
\hline Variável & Condição & N & $\%$ \\
\hline Sexo & Masculino & 358 & 55,3 \\
& Feminino & 289 & 44,7 \\
Faixa etária & $20-29$ & 83 & 12,8 \\
& $30-39$ & 295 & 45,6 \\
& $40-49$ & 246 & 38,0 \\
& $50-59$ & 17 & 2,6 \\
Escolaridade & Até 2o grau completo & 87 & 14,0 \\
& Mais que 2o grau & 545 & 86,0 \\
Situação & Casados & 391 & 60,4 \\
conjugal & Solteiros & 161 & 24,9 \\
& Divorc/separados/viúvos & 86 & 13,3 \\
Imóvel & Próprio (quitado ou não) & 393 & 60,4 \\
& De alguém da & & \\
& família/emprestado & 133 & 20,5 \\
& Alugado & 108 & 16,7 \\
\hline
\end{tabular}

nas), $86,4 \%$ não tinham contato com o público. Além disso, cerca da metade dos funcionários tinha função comissionada, que, em geral, representa cargo de maior responsabilidade.

A prevalência de alguns dos principais fatores de risco para doenças cardiovasculares (Tabela 2) mostra que, exceto para prática de atividades físicas regulares, os demais fatores de risco apresentaram maiores prevalência entre os homens, quando comparados às mulheres. Cabe destacar que as diferenças mais expressivas entre homens e mulheres foram observadas nas prevalências de sobrepeso e obesidade e da ingesta de bebidas alcoólicas.

A prevalência de tabagismo entre os funcionários foi de $29,5 \%$ (IC 95\%:27,5\% a 31,5\%), sem diferença significativa entre os sexos (Tabela 3). Entre fumantes e ex-fumantes, as idades de início do hábito de fumar variaram de 11 a 34 anos, sendo que $46 \%$ dos funcionários iniciaram até os 17 anos. A média de idade de início do hábito foi de 17,6 anos entre os homens e 19,4 entre as mulheres ( $p<0,001)$, com moda de 16 e 18 anos, respectivamente.

O consumo diário de cigarros pode ser considerado elevado em ambos os sexos, já que 53\% dos homens e $42 \%$ das mulheres relataram consumir mais de 20 cigarros por dia (Tabela 4). A média de consumo diário de cigarros foi 19,6 entre os homens e 16,7 entre as mulheres $(\mathrm{p}=0,557)$.

Com relação à restrição do uso do tabaco na empresa, a maioria ( $88 \%$ ) dos funcionários responderam que era permitido fumar no ambiente do trabalho.

Tabela 2 - Prevalência de fatores de risco entre a população estudada, 1994.

\begin{tabular}{|c|c|c|c|c|c|c|}
\hline \multirow{2}{*}{ Fatores de risco } & \multicolumn{2}{|c|}{ Homens } & \multicolumn{2}{|c|}{ Mulheres } & \multicolumn{2}{|c|}{ Total } \\
\hline & $\mathrm{N}$ & $\%$ & $\mathrm{~N}$ & $\%$ & $\mathrm{~N}$ & $\%$ \\
\hline Hipertensão arterial & 63 & 18,2 & 32 & 11,3 & 95 & 14,7 \\
\hline IMC (peso/altura $\left.{ }^{2}\right) \geq 25 \mathrm{~kg} / \mathrm{m}^{2}$ & 173 & 50,8 & 62 & 23,5 & 235 & 36,3 \\
\hline Informado sobre colesterol elevado & 70 & 19,8 & 44 & 15,3 & 114 & 17,6 \\
\hline N ão praticam atividades físicas & 202 & 56,4 & 173 & 59,9 & 375 & 58,0 \\
\hline Bebem freqüentemente & 194 & 54,2 & 75 & 26,0 & 269 & 41,6 \\
\hline
\end{tabular}

IMC - Índice de massa corporal

Tabela 3 - Prevalência de tabagismo segundo sexo entre a população estudada,1994.

\begin{tabular}{lrrrrr}
\hline \multirow{2}{*}{ U so do tabaco } & \multicolumn{2}{c}{ Homens } & \multicolumn{2}{c}{ Mulheres } \\
\cline { 2 - 5 } & \multicolumn{1}{c}{$\mathrm{N}$} & $\%$ & $\mathrm{~N}$ & $\%$ \\
\hline Fumantes & 111 & 31,1 & 80 & 27,8 \\
Ex-fumantes & 78 & 21,8 & 65 & 22,6 \\
Não-fumantes & 168 & 47,1 & 143 & 49,7 \\
& & & & \\
\hline Total & 357 & 100,0 & 288 & 100,0 \\
\hline$\left(\chi^{2}=0,85 ; p=0,653\right)$ & & & &
\end{tabular}

Tabela 4 - Q uantidade de cigarros consumidos diariamente segundo sexo entre a população estudada, 1994.

\begin{tabular}{lrrrr}
\hline \multicolumn{1}{c}{ Cigarros/dia } & \multicolumn{2}{c}{ Homens } & \multicolumn{2}{c}{ Mulheres } \\
\hline & $\mathrm{N}$ & $\%$ & $\mathrm{~N}$ & $\%$ \\
Até 9 & 46 & 25,6 & 46 & 34,8 \\
10 a 19 & 38 & 21,1 & 30 & 22,7 \\
20 a 39 & 79 & 43,9 & 50 & 37,9 \\
40 e + & 17 & 9,4 & 6 & 4,5 \\
\hline Total & 180 & 100,0 & 132 & 100,0 \\
\hline$\left(\chi^{2}=5,46 ; p=0,140\right)$ & & & &
\end{tabular}


Através de análise multivariada foi possível identificar associação entre tabagismo e os seguintes fatores: idade (os mais velhos tinham maior chance de serem fumantes); estado conjugal (divorciados, separados e viúvos tinham maior chance de serem fumantes do que os casados); hipertensão arterial; consumo de álcool; e ausência de atividades físicas regulares (Tabela 5).
Para ex-fumantes, as associações observadas foram para idade (a chance de deixar de fumar aumentou com a idade); situação conjugal (solteiros tinham menor chance de serem ex-fumantes do que os casados); consumo do álcool e estado nutricional (exfumantes tinham maior chance de apresentarem sobrepeso e obesidade do que os não-fumantes) (Tabela 6).

Tabela 5 - Associação bruta e ajustada entre tabagismo atual e variáveis selecionadas no modelo de regressão logística, 1994.

\begin{tabular}{|c|c|c|c|c|}
\hline \multirow{2}{*}{ Variáveis } & \multicolumn{2}{|c|}{ Razão de chances ajustadas } & \multicolumn{2}{|c|}{ Razão de chances brutas } \\
\hline & \multicolumn{2}{|c|}{$(\mathrm{IC} 95 \%)$} & \multicolumn{2}{|c|}{$(\mathrm{IC} 95 \%)$} \\
\hline \multicolumn{5}{|l|}{ Idade } \\
\hline$<40$ anos & 1,00 & & 1,00 & \\
\hline$>=40$ anos & 1,82 & $(1,18-2,81)$ & 1,56 & $(1,08-2,26)$ \\
\hline \multicolumn{5}{|l|}{ Sexo } \\
\hline Feminino & 1,00 & & 1,00 & \\
\hline Masculino & 1,18 & $(0,75-1,84)$ & 1,18 & $(0,82-1,69)$ \\
\hline \multicolumn{5}{|l|}{ Situação conjugal } \\
\hline Casados & 1,00 & & 1,00 & \\
\hline Solteiros & 0,97 & $(0,60-1,57)$ & 0,88 & $(0,57-1,34)$ \\
\hline Divorc/separ/viúvos & 2,85 & $(1,52-5,32)$ & 2,24 & $(1,29-3,88)$ \\
\hline \multicolumn{5}{|l|}{ H ipertensão arterial } \\
\hline Não & 1,00 & & 1,00 & \\
\hline Sim & 2,21 & $(1,23-3,99)$ & 1,91 & $(1,14-3,21)$ \\
\hline \multicolumn{5}{|l|}{ Freqüência utiliz. álcool } \\
\hline $\mathrm{N}$ ão bebem & 1,00 & & 1,00 & \\
\hline Bebem ocasionalmente & 4,40 & $(1,48-13,05)$ & 2,14 & $(1,02-4,48)$ \\
\hline Bebem freqüentemente & 10,92 & $(3,64-32,75)$ & 4,23 & $(2,02-8,85)$ \\
\hline \multicolumn{5}{|l|}{ Atividades físicas } \\
\hline $\mathrm{N}$ ão pratica & 1,00 & & 1,00 & \\
\hline Pratica & 0,47 & $(0,31-0,73)$ & 0,57 & $(0,39-0,83)$ \\
\hline
\end{tabular}

Tabela 6 - Associação bruta e ajustada entre deixar de fumar e variáveis selecionadas no modelo de regressão logística, 1994

\begin{tabular}{|c|c|c|c|c|}
\hline \multirow{2}{*}{ Variáveis } & \multicolumn{2}{|c|}{ Razão de chances ajustadas } & \multicolumn{2}{|c|}{ Razão de chances brutas } \\
\hline & \multicolumn{2}{|c|}{$(\mathrm{IC} 95 \%)$} & \multicolumn{2}{|c|}{ (IC 95\%) } \\
\hline \multicolumn{5}{|l|}{ Idade } \\
\hline$<40$ anos & 1,00 & & 1,00 & \\
\hline$>=40$ anos & 2,12 & $(1,32-3,41)$ & 1,98 & $(1,32-2,98)$ \\
\hline \multicolumn{5}{|l|}{ Sexo } \\
\hline Feminino & 1,00 & & 1,00 & \\
\hline Masculino & 1,00 & $(0,59-1,71)$ & 1,02 & $(0,86-1,52)$ \\
\hline \multicolumn{5}{|l|}{ Situação conjugal } \\
\hline Casados & 1,00 & & 1,00 & \\
\hline Solteiros & 0,33 & $(0,17-0,65)$ & 0,41 & $(0,24-0,70)$ \\
\hline Divorc/separ/viúvos & 1,73 & $(0,82-3,62)$ & 1,46 & $(0,79-2,70)$ \\
\hline \multicolumn{5}{|l|}{ Freqüência utiliz. álcool } \\
\hline $\mathrm{N}$ ão bebem & 1,00 & & 1,00 & \\
\hline Bebem ocasionalmente & 8,21 & $(1,86-36,22)$ & 2,29 & $(1,06-4,93)$ \\
\hline Bebem freqüentemente & 9,08 & $(2,01-40,88)$ & 2,69 & $(1,23-5,90)$ \\
\hline \multicolumn{5}{|l|}{ Estado nutricional } \\
\hline Normal & 1,00 & & 1,00 & \\
\hline Sobrepeso & 1,74 & $(1,02-2,97)$ & 1,71 & $(1,08-2,71)$ \\
\hline O besidade & 3,04 & $(1,34-6,90)$ & 2,66 & $(1,30-5,42)$ \\
\hline
\end{tabular}




\section{DISCU SSÃO}

O questionário auto-respondido apresentou vantagens, que se referem não só à diminuição de custos e do tempo necessário para desenvolver a pesquisa, mas também pareceu ideal naquele ambiente de trabalho, uma vez que os funcionários responderam durante o expediente, no momento mais disponível, sem prejuízos das suas funções. Por outro lado, o questionário respondido por eles próprios, com a garantia do sigilo das informações, além de todos possuírem estabilidade na empresa, pode ter incentivado respostas mais sinceras e evitado constrangimentos quanto a informar problemas de saúde e hábitos socialmente indesejáveis. Acredita-se que esses fatores tenham contribuído também para a grande adesão à pesquisa.

A importância do estudo do hábito de fumar e seus determinantes não se restringe a este comportamento mas também pode ser indicação da ocorrência de diversos outros considerados de risco para as doenças cardiovasculares. Nesse sentido, diversas investigações concluíram que hábitos não saudáveis ocorrem juntos ("em cluster") desde a infância e adolescência até a vida adulta (Raitakari ${ }^{32}$, 1995; Jousilahti ${ }^{21}$, 1994). Chor ${ }^{5}$ (1997) identificou que grandes fumantes (mais de 20 cigarros diários por dia) apresentavam maior probabilidade de consumirem álcool diariamente, serem sedentários e terem dieta rica em gordura saturada, colesterol e sal na mesma empresa desta investigação. $\mathrm{O}$ grupo examinado, apesar de sua especificidade, pode também dar indicativos do estilo de vida de uma categoria numerosa de adultos jovens e indivíduos de meia-idade que habitam as grandes metrópoles brasileiras, trabalham na área de serviços e pertencem à classe média.

A prevalência de fumantes do sexo masculino $(31,1 \%)$ foi inferior às estimativas de outras pesquisas nacionais, enquanto a proporção de mulheres $(27,8 \%)$ foi semelhante. Na região de Porto Alegre, Moreira e col. ${ }^{27}$ (1995) encontrou 41,5\% entre os homens e $29,5 \%$ entre mulheres. Em doze capitais brasileiras, as prevalências foram $45 \%$ entre os homens e 33\% entre as mulheres (Minitério da Saú$\left.\mathrm{de}^{25}, 1992\right)$. Esses estudos abrangeram populações em que diversos estratos sociais estavam representados, enquanto os funcionários da empresa estudada constituíram grupo mais homogêneo, mais favorecido na escala social e com maior nível de escolaridade, o que justifica a menor prevalência entre os homens (Chor ${ }^{5}$, 1997).
Os dados encontrados parecem confirmar a tendência mundial de aproximação da prevalência de fumantes entre os sexos, através de seu declínio entre os homens e do aumento ou estabilização entre mulheres (Epstein $\left.{ }^{10}, 1989\right)$. Por outro lado, assemelham-se aos dados de Blaxter ${ }^{2}$ (1990) que demonstrou na população inglesa pequena diferença de prevalência de fumantes entre os sexos para classes sociais mais elevadas, o que não ocorreu entre os mais pobres, entre os quais observou-se maior prevalência entre os homens. Entre os funcionários dos CESECs, a diferença na prevalência de tabagismo entre os sexos não foi estatisticamente significativa. Assim, o padrão encontrado entre os bancários parece acompanhar aquele de países desenvolvidos, onde a diferença entre homens e mulheres é pequena. Nos Estados Unidos, em 1987, estimou-se $32 \%$ entre os homens e $27 \%$ entre as mulheres (Novotny e col. $\left.^{28}, 1990\right)$; e no Canadá, em 1983, 31\% e 28\%, respectivamente (Epstein $\left.{ }^{10}, 1989\right)$. Além disso, o consumo médio diário de cigarros não foi diferente entre homens e mulheres.

Também entre os funcionários da empresa estudada o hábito de fumar iniciou-se principalmente na adolescência, conforme já descrito em outros estudos (Elders e col. ${ }^{8}$, 1994; Ena ${ }^{9}$, 1993), reforçando a importância de estratégias de controle de propagandas e marketing sobre o cigarro, dirigidas a esta faixa etária.

No banco estudado, têm sido feitos investimentos na promoção da saúde dos seus funcionários, principalmente em campanhas antitabagistas ${ }^{26}$. Porém, os presentes dados indicam que ainda existem oportunidades perdidas no controle do que constitui um dos mais importantes fatores de risco para doenças crônicas, já que a grande maioria dos trabalhadores declarou que o tabagismo é permitido na empresa. Segundo Silva e col. ${ }^{37}$ (1993), as doenças do aparelho respiratório foram apontadas como primeiro motivo de licenças entre os funcionários dos CESECs, em 1992, perfazendo $27,4 \%$ do total. Outro estudo de mortalidade ${ }^{16}$ apontou excesso de mortalidade por câncer de pulmão entre os homens do mesmo banco estudado, quando comparadas às taxas dos homens de São Paulo. No entanto, não foi possível identificar a influência do tabaco nesses achados, principalmente dos malefícios agravados pelos ambientes fechados e mantidos permanentemente sob refrigeração artificial. Mesmo que esta pesquisa não indique elementos que reforcem ou inibam o consumo de cigarros no ambiente de trabalho, já existe um corpo de conhecimentos razoavelmente estabelecidos que justificam ações persuasi- 
vas e coercitivas visando a reduzir a poluição de ambientes pelo uso de tabaco.

Os dados encontrados na análise multivariada apontaram forte associação entre tabaco e uso do álcool, já relatada em outros estudos (Woodward e col. ${ }^{42}$, 1994; Kabat e col. ${ }^{22}$, 1994), sugerindo determinantes comuns (Moreira e col. $\left.{ }^{27}, 1995\right)$. Semelhante ao estudo de Thornton e Fry ${ }^{40}$ (1994), a presente pesquisa também apontou maior proporção de fumantes entre os divorciados, separados e viúvos. Os mesmos autores também encontraram que os fumantes praticam menos atividades físicas regulares do que os não-fumantes, como entre os bancários. Porém, diferente de outros estudos, que não avaliaram populações tão específicas como no caso desta investigação (Klein e Araújo ${ }^{24}$, 1985; Woodward e col. ${ }^{42}$, 1994; Thornton e Fry ${ }^{40}$, 1994; Fuchs e col. ${ }^{14}$, 1995), foram encontrada associação entre hipertensão e uso do tabaco, sinalizando que, o alto nível de escolaridade, o acesso aos serviços de saúde e à informação não estão sendo suficientes para mudanças de hábitos e comportamentos visando ao controle das doenças cardiovasculares (Chor5 , 1997).

Em geral, indivíduos que param de fumar ganham peso subseqüentemente (Swan e Carmelli ${ }^{38}$, 1995). No presente estudo, foram detectadas altas prevalências de sobrepeso e obesidade entre ex-fumantes. Apesar de não ser possível saber se o aumento de peso ocorreu antes ou após deixarem de fumar, seria interessante atentar mais para este gru- po no sentido de investigar, de forma mais detalhada, a presença de outro importante fator de risco para doenças cardiovasculares. O mesmo destaque deve ser dado ao consumo de bebidas alcóolicas, que se mostrou bastante elevado entre funcionários que deixaram de fumar quando comparados aos que nunca fumaram.

Sabe-se que o que tem levado a maioria dos países desenvolvidos a criar programas comunitários ou nas empresas buscando a redução de fatores de risco para doenças cardiovasculares, é principalmente a possibilidade de reverter, tanto a prevalência dos mesmos, como a morbi-mortalidade a eles associada (Gomel e col. $\left.{ }^{16}, 1993\right)$. Entre os bancários, o tabagismo é certamente um hábito passível de modificação. Assim, são essenciais as campanhas educativas no que diz respeito à mudança no estilo de vida dos funcionários dos CESECs, não só referentes ao tabagismo, mas em relação a todos os fatores de risco para doenças cardiovasculares A concepção de "estilo de vida" no presente trabalho, é a que se constitui em hábitos e comportamentos socialmente determinados, e não são uma questão apenas de responsabilidade individual. Nesse sentido, o ambiente de trabalho, onde os adultos passam a maior parte do tempo, é um lugar onde estes hábitos podem ser reforçados ou modificados, representando um enorme potencial para programas de prevenção. A aplicação de recursos nesses programas costuma ter relação custo-benefício mais favorável quando comparado com serviços de atendimento.

\section{REFERÊNCIAS}

1. BAAN, B. Prevention of smoking in young children in Holland: education and changing attitudes. Lung (Supll.):320-6, 1990.

2. BLAXTER, M. Health and lifestyles. London, Routledge, 1990.

3. BRIGHAM, J. et al. Effects of a restricted work-site smoking policy on employees who smoke. Am. J. Public Health, 84:773-8, 1994.

4. CAWSTON, P. \& McEWEN, J. Three-year follow-up survey of smokers who attended "Good Hearted Glasgow" sreening sessions. Public Heatlh, 108: 185-94, 1994.

5. CHOR, D. Perfil de risco cardiovascular de funcionários de Banco Estatal. São Paulo, 1997. [Tese de Doutorado Faculdade de Saúde Pública da USP].

6. DAUGHTON, D.M. et al. Smoking cessation in the workplace evaluation of relapse factors. Prev. Med., 19:227-30, 1990.
7. DOLL, R. \& HILL, B. Lung cancer and other causes of death in relation to smoking. Br. Med. J., 10:1071-81, 1956.

8 ELDERS, M.J. et al. The Report of the Surgeon General: preventing tobacco use among young people. Am. J. Public Health, 84:543-7, 1994.

9. ENCUESTA NACIONAL DE ADICCIONES (ENA). Información epidemiológica. México, 1993.

10. EPSTEIN, F. H. The relationship of lifestyle to international trends in CHD. Int. J. Epidemiol., 18:511-7, 1989.

11. FIELDING, J. et al. Health promotion programs sponsoed by California employers. Am. J. Public Health, 73:538-42, 1993.

12. FIELDING, J. et al. Worksite health promotion survey: smoking control activities.Prev. Med., 19: 402-13, 1990.

13. FIELDING, J. Smoking: health effects and control. In: Fielding, J. Maxcy Rosenau public health and preventive medicine. Aplleton \& Lange, 1986. p. 999-1038. 
14. FUCHS, F.D. et al. Prevalência de hipertensão arterial sistêmica e fatores associados na região urbana de Porto Alegre: estudo de base populacional. Arq. Bras. Cardiol., 63: 473-9, 1995.

15. FUNDAÇÃO IBGE. Pesquisa nacional sobre saúde e nutrição; dados Preliminares. Rio de Janeiro, 1989.

16. GOMEL, M. et al. Pilot study of the effects of a workplace smoking ban on indices of smoking, cigarette craving, stress and other health behaviours. Psychol. Health, 8: 223-9, 1993.

17. GRUPOTÉCNICO CASSI PESQUISAS EPIDEMIOLÓGICAS. Estudo de mortalidade dos funcionários do Banco do Brasil; Relatório Final,1992.

18. HOPPENBROUWERS, T. Airways and air pollution in childhood: state of the art. Lung (Supll.):335-46, 1990.

19. JEFFERY, R. et al. Restrictive smoking policies en the workplace: effects on smoking prevalence and cigarette consumption. Prev. Med., 23: 78-82, 1994.

20. JOOSENS, L. Smoking policy in the workplace and other public places. Lung (Supll.):437-44,1990.

21. JOUSILAHTI, P. et al.Trens in cardiovascular disease risk factors clustering in eastern Finland: results of 15-year follow-up of the North Karelia Project. Prev. Med., 23: 6-14, 1994.

22. KABAT, G. C. et al. The role of tobacco, alcohol use, and body mass index in oral and pharyngeal cancer. Int. J. Epidemiol., 23:1137-45, 1994

23. KAVANAGH, D. J. et al. Self-efficacy and social support as predictors of smoking after a quit attempt. Psychol. Health, 8:231-42, 1993

24. KLEIN, C. H. \& ARAÚJO, J. W.G. Fumo, bebida alcoólica, migração, instrução, ocupação, agregação familiar e pressão arterial em Volta Redonda, Rio de Janeiro. Cad. Saúde Pública, 2:160-76, 1985.

25. MINISTÉRIO DA SAÚDE. Atualidades em tabagismo. Rio de Janeiro, Instituto Nacional de Câncer, Coordenação de Programas de Controle de Câncer, 1993.

26. MINISTÉRIO DA SAÚDE. Controle do tabagismo: um desafio. Rio de Janeiro. Instituto Nacional de Câncer, Coordenação de Programas de Controle de Câncer, 1992.

27. MOREIRA, L.B. et al. Prevalência de tabagismo e fatores associados em área metropolitana da região Sul do Brasil. Rev. Saúde Pública, 29:46-51, 1995.
28. NOVOTNY, T. et al. Trends in smoking by age and sex, United States, 1974-1987: the implications for disease impact. Prev. Med.,19:552-61, 1990.

29. OSLER, M. Social class and health behaviour in Danish adults: a longitudinal study. Public Health, 107:251-60, 1993.

30. PRICE, J. A. Nonpharmacologic means of preventing asthma. Lung (Supll.):286-91, 1990

31. PUSKA, P. et al. Change in risk factors for coronary heart disease during 10 years of a community intervention programme (North Karelia Project). Br. Med. J., 287:1840-4, 1983

32. RAITAKARI, O. T. Clustering of risk habits in young adults: the cardiovascular risk in young finns study. Am. J. Epidemiol., 142:36-44, 1995.

33. ROEMER, R. Legislative action to combat the world tobacco epidemic. 2nd ed. Geneva, World Health Organization, 1993

34. RONCHETTI, R. et al. Passive smoking in childhood tobacco smoke. Lung (Supll.):313-9, 1990.

35. ROSEMBERG, J. Tabagismo: sério problema de saúde pública. São Paulo, ALMED, 1987.

36. SERXNER, S. et al. Influences on cigarette smoking quantity. J. Occup. Med., 34:934-9, 1992.

37. SILVA FILHO, J .F. da et al. Organização do trabalho e saúde mental: estudo das relações entre a prevalência de doenças mentais e organização do trabalho bancário no Município do Rio de Janeiro (RJ). Rio de Janeiro. Convênio FUJB/FBB, Relatório de Pesquisa, 1993.

38. SWAN, G. E. \& CARMELLI, D. Characteristics associated with excessive weight gain after smoking cessation in men. Am. J. Public Health, 85: 73-7, 1995.

39. TAYLOR, B. Prevention of pediatric pulmonary problems: the importance of maternal smoking. Lung (Supll.):327-32, 1990.

40. THORNTON, P. L. \& FRY, J. Differences between smokers, ex-smokers, passive smokers and non-smokers. J. Clin Epidemiol., 47: 1143-62,1994.

41. WAKEFIELD, M. A. et al. Workplace smoking restrictions, occupational status, and reduced cigarette consumption. JOM, 34:693-7, 1992.

42. WOODWARD, M. et al. Dedicient health knowledge, diet, and other lifestyles in smokers: is a multifactorial approach required? Prev. Med., 23: 354-61,1994. 